\title{
Real-Time Monitoring of Patients with Coronary Artery Disease
}

\author{
Ahmed F. Otoom, Ahmed Kefaye, Mohammad Ashour, Yousef Shanti, and Mohammad Al-Majali
}

\begin{abstract}
The emergence of wearable sensor mobile technologies can have a great change to the conventional healthcare systems improving the quality of life for patients with Coronary Artery Disease (CAD) or heart disease. Our main aim is to build a simple yet accurate mobile application that is capable of real-time monitoring of patients with CAD which is a major cause of death worldwide. Most available mobile healthcare systems focus on the data acquisition part with little attention paid to facilitating real-time. In this work, we build a real-time monitoring application that continuously monitors CAD patients and raises an alarm whenever an emergency occurs. The proposed system is of a light weight, low price and very reliable in the detection of heart problems based on reasoning and computational algorithms. To prove the effectiveness of the proposed system, we conducted two extensive experiments for real and simulated scenarios. Our results show that the proposed monitoring system provides a $100 \%$ detection rate.
\end{abstract}

Index Terms-Coronary artery disease, heart disease monitoring, wearable sensors, mobile application.

\section{INTRODUCTION AND RELATED WORK}

Recently, there has been growing interest in wearable health monitoring devices, both in research and industry. These devices are important for patients who need to be assessed regularly or monitored continuously [1]. They are vital to decrease the numbers of people visiting hospitals especially for those with complex medical conditions. Across the globe, there is increasing numbers of population putting stress on the current health care systems that are not structured to service rising needs of high numbers of patients [2]. Current advances in the areas of computer, networking and medical fields have brought together smart health care options that depend on mobile phones and wearable sensors which are now accessible for many people worldwide with affordable prices.

According to the American Heart Association (AHA), heart disease, also referred to as coronary artery disease $(\mathrm{CAD})$ is a term used to describe a variety of problems related to plaque buildup in the walls of arteries making it gradually

Manuscript received January 12, 2015; revised March 25, 2015.

A. F. Otoom is with the Prince Al-Hussein Bin Abdullah II for IT, The Hashemite University, Jordan (e-mail: aotoom@hu.edu.jo).

A. Kefaye is with Alpha-Hub Company, Jordan (e-mail: ahmad@alpha-hub.com).

M. Ashour is with Telelogx Company, Jordan (e-mail: m.ashour@teleogx.com).

Y. Shanti is with UXBERT Company, Saudi Arabia (e-mail: m.ashour@uxbert.com).

M. Al-Majali is with Niche Marketing Management Organization, UAE (e-mail: almajali@gmail.com). narrow and in consequence it becomes difficult for blood to flow increasing the risk of heart attack and stroke [3].

In 2010, CAD was a major cause of death worldwide and was responsible for one in every nine deaths in the United States [3]. In some cases, people with heart disease may have symptoms like chest pain and fatigue. However, in many cases, there are no symptoms until a heart attack occurs [4]. This was the major motive for us to build a smart system that can continuously monitor the person's heart and raise attention whenever there is any heart related problem.

The adoption of mobile health care applications is promising to enhance the quality of life for patients with CAD. With these applications, the patients can have a more independent and easy life and a quick action can be triggered which is crucial as few minutes can save lives for people with heart disease [5]. In addition, with many patients living in rural areas and with a reduced access to health providers, the development of such systems can help with continuous remote monitoring for these people with a reduced number of visits to hospitals. Moreover, the cost of remote health monitoring systems is affordable to a wide range of people because of the recent advances in consumer electronics and mobile technologies which have reduced the production costs and have made it possible to ordinary users to afford inexpensive sensors and mobile phones [5].

A central part of any monitoring systems is the use of wearable sensors which would allow people to be constantly monitored. Wearable sensors can be defined as electronic devices that can be unremarkably embedded in the user's outfit as part of the clothing or an accessory. They range from micro-sensors to computerized watches and belt-worn PCs with a head mounted display [6]. Wearable sensors have many applications that include physiological, biochemical, and motion sensing [7].

Sensors are deployed for patients with heart problems for continuous monitoring. For example, in the work of [8], a ring sensor worn on the base of the finger is implemented for the purpose of continuous monitoring for people with congestive heart failure problems. Another example is presented in the work of [9] where an energy efficient ear worn PPG sensor is introduced for the purpose of heart monitoring. The comfortable placement of the sensor makes it suitable for long term monitoring, often referred to this feature as mobility. Two major advantages of the abovementioned examples is the small size of the sensors and the low power consumption which are very important issues to be considered in any monitoring problem. Recently, the authors in [10] proposed an Android-based system for heart monitoring using data collected from a strap worn around the chest named Zephyr Heart Rate Monitor. It measures heart rate and other 
measurements and uses Bluetooth to send the information to an Android phone device. The measurements are used to identify a number of heart related problems. The system proved useful and the authors achieved good performance results.

It is important to note that health monitoring applications of wearable systems often employ multiple sensors that are integrated together to form a body sensor network. An example of these applications is MyHeart [11], where a number of on-body sensors are used to collect physiological data that are sent wirelessly to a PDA. The information is analyzed and health recommendations are given to the user based on this analysis.

Once data is collected, wireless communication is then relied upon to transmit patients' data to a mobile phone or an access point and relay the information to a remote center via the internet. Emergency situations are detected via data processing implemented throughout the system and an alarm message is sent to an emergency service center to provide direct assistance to patients [7].

Most available commercial applications have focused on the data acquisition part with little attention paid to facilitating real-time. The proposed system offers real time application that can continuously monitor the user and raise an alarm for any emergency caused by the detection of a heart problem. In addition, the proposed wearable health monitoring system is of a light weight, low price and very reliable in the detection of heart problems based on reasoning and computational algorithms.

The rest of this paper is organized as follows: In Section II, we explain in detail the components of the system. Section III presents the experimental results of the proposed application. Finally, Section IV concludes the paper and suggests future work.

\section{SYSTEM DESIGN}

Although there are many commercially available healthcare systems, they usually share a common prototype [5]. For heart monitoring systems, this prototype usually consists of a sensor carried by the patient that is connected to a backbone network via a gateway node. The patient and doctors can monitor heart rate information by a graphical user interface (GUI). In the case of an emergency situation, an alert is sent to a laptop or a personal computer in the closest hospital.

Our proposed application consists of three main components: i) data acquisition, ii) a gateway to the wide area networks (WANs), and iii) the end user health care application. In this section we will discuss each component in details (see Fig. 1 for a conceptual representation of the proposed system).

\section{A. Data Acquisition}

To detect the heart rate, we implement a commercially available sensor named, the Pulse Sensor AMPED [12]. It combines a simple optical heart rate sensor with amplification and noise cancellation circuitry making it fast and easy for getting reliable heart pulse readings.

This specific sensor was chosen because of its reliability, high usability and good performance. Moreover, it is of a small size and a modest price. The sensor can be simply worn by clipping it to the finger tip and connecting it to a microcontroller.

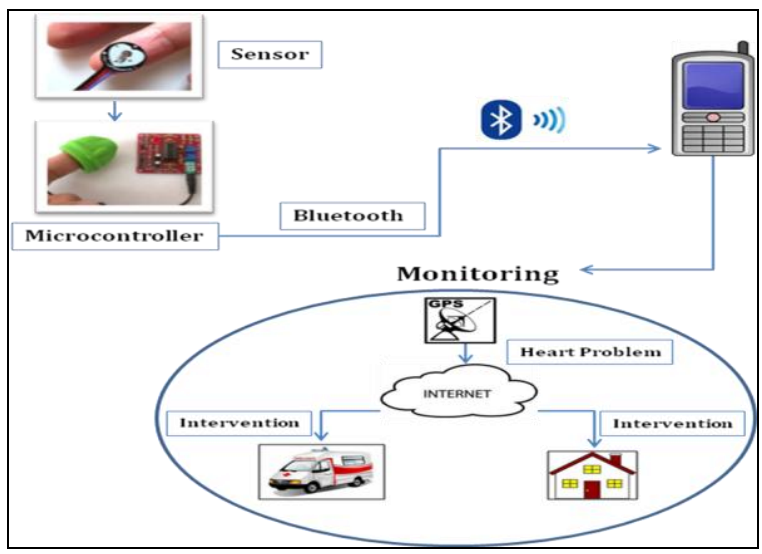

Fig. 1. A conceptual representation of the proposed system

An important issue to consider when implementing sensors is power consumption. The implemented sensor is energy efficient with a power consumption of only $\sim 4 \mathrm{~mA}$ at $5 \mathrm{~V}$ with voltage ranging from $3 \mathrm{~V}$ to $5 \mathrm{~V}$. Moreover, the low output transmission power of the sensor is important for keeping its effects minimal on the patient's health.

\section{B. Gateway to Wide Area Networks (WANs)}

This component is responsible for connecting the sensing component to the infrastructure based WANs. In other words, data collected from the sensor should be transmitted through a gateway, which is usually a mobile phone or a personal computer, to a remote site such as a hospital.
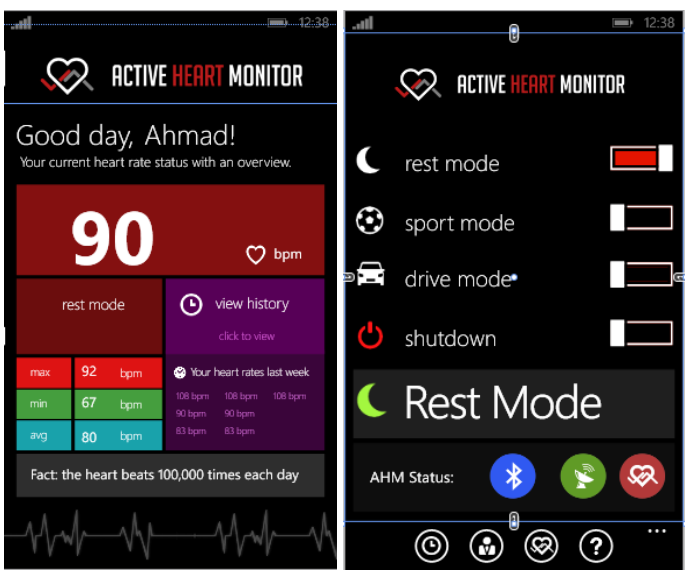

Fig. 2. Interfaces from the proposed mobile application.

In our application, this connection is carried out by firstly connecting the sensor to an arduino microcontroller and then the sensing data is wirelessly transmitted through a Bluetooth technology to a mobile phone. The Bluetooth module that is integrated in the arduino microcontroller is HC-06 module which uses the UART (Universal asynchronous receiver/transmitter) protocol. UART makes it easy and reliable to send and receive data wirelessly.

In case of an emergency situation which is identified based on the data analysis on the mobile device, an emergency alert is relayed to the closest hospital through the Internet. The location of the closest hospital is identified using GPS technology which is integrated with the majority of mobile 
phones currently available with people worldwide.

As the information is relayed to the hospital through a network system, the Internet, it is crucial to have reliable communication protocols for wide area networks. A universal broadband connectivity is very important to achieve such reliability. Broadband technologies are currently available in the majority of the developed countries and across many developing countries [7]. Moreover, a reliable Bluetooth module for accurate transmission of data from the microcontroller to the mobile device is implemnted and it has proved to be effective during testing.

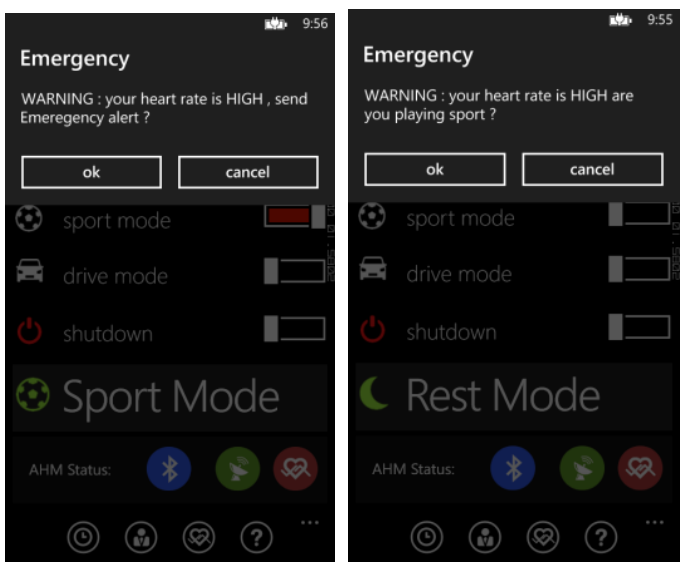

Fig. 3. Emergency alerts.

\section{The End User Application}

The end user application is a central part of the system where the collected data are analyzed and actions are triggered [5], (see Fig. 2 for two screens of the application interface). The application performs reasoning with computational algorithms to continuously monitor the patient's heart rate and save all the readings of the heart rates (Beats Per Minute (BPM)) to a log file. This saved data can be reviewed when needed, e.g. when the patient visits his/her health clinic.

Once an emergency situation occurs, the algorithm detects it and automatically generates an email that contains the patient's information from name, mobile phone, age, and the exact location based on the GPS coordinates. An emergency SMS is also generated. The email is sent to the closest hospital to the patient's location. The closest hospital is identified using the GPS technology. The location of the user is updated every 1 minute. Moreover, the emergency SMS alert is sent to the patient's relative together with the exact location of the patient. According to the American Heart Association, most people have a resting heart rate of 60 to 100 beats per minute. For people playing sports, this heart rate may increase while playing to reach values of up 150 BPM. We adopted this criterion in our system, and our algorithm raises an alarm whenever the heart rate deviates from the aforementioned rates.

The proposed application implements two modes: the rest mode and the sport mode which are set by the user. If the person is in the rest mode and the heart rate deviates from its specified thresholds, the system waits for window interval of 5 minutes and during this time, if the heart rate remains unstable, the system notifies the user whether he/she is playing sport or not. If the user is playing sports, then the system changes to the sport mode and if not, it sends an emergency alert. Similarly, in the sport mode, if the monitoring system detects an emergency status that remains for a window interval of 5 minutes, it sends a notification email and an SMS alert to the closest hospital and the patient's relative, respectively. The window interval is inspired by works like [10] and based on consultations with a number of medical experts. Fig. 3 shows examples for screens of the emergency alerts. In addition, a driving mode can be also be set for a continuous display of BPM on the vehicles windshield making it easier for the driver to monitor his/her heart rate.

A prototype for a diagnosis component is also developed in this work. The interfaces are designed to accept a number of inputs that can be entered by the user or the doctor. These inputs correspond to features implemented in the Heart data set available from the UCI Machine Learning Repository [13]. The data set consists of 303 individuals' clinical records of which 164 did not have heart disease. This data set can be used with machine learning techniques to build a smart classifier for a prediction of the existence of CAD. The prototype facilitates a future integration of a diagnosis component.

\section{EXPERIMENTAL RESULTS AND ANALYSIS}

Experiments are conducted in order to evaluate the performance of the proposed system. We carried out these experiments using Nokia lumia 520 mobile phone. It is equipped with a Duel-core $1 \mathrm{GHz}$ qualcomm snapdragon $\mathrm{S} 4$ processor, an internal 8 GB storage and it includes a Bluetooth $v 4.0$ interface. To evaluate the performance of the proposed application, we carried out trial experiments involving 20 healthy individuals where 5 of them were asked to play sport while carrying the monitoring device. As all the selected personnel were healthy, there were no deviations from the specified thresholds and thus no alerts generated. Table 1 shows the results of the heart monitoring test with the selected sample. It is clear from this table that the algorithm is $100 \%$ accurate in the monitoring component for both modes and with no generated false alarms. Moreover, the BPM detected values are comparable to real values. These are interesting results and prove the usefulness of the proposed monitoring system.

TABLE T: MONITORING RESULTS

\begin{tabular}{ccc}
\hline Test Case & $\begin{array}{c}\text { \# of } \\
\text { samples }\end{array}$ & $\begin{array}{c}\text { detection } \\
\text { results }\end{array}$ \\
\hline Healthy / Rest mode & 15 & $100 \%$ \\
\hline Healthy / Sport mode & 5 & $100 \%$ \\
\hline TABLE II: MonITORING RESULTS WITH SIMULATED CASES \\
\hline Test Case & $\begin{array}{c}\text { \# of } \\
\text { samples }\end{array}$ & $\begin{array}{c}\text { detection } \\
\text { results }\end{array}$ \\
\hline Patients / Rest mode (simulated) & 10 & $100 \%$ \\
\hline Patients / Sport mode (simulated) & 10 & $100 \%$ \\
\hline
\end{tabular}

To further test the performance of the proposed system for heart disease patients, we used offline implementation that 
simulates real time device operation for 20 unhealthy cases where both the rest mode and sport mode were tested. Deviations from normal rates for both modes were simulated with different values that are more and less than the specified thresholds. The monitoring system accurately detected these deviations and emergency alerts and SMSs were generated in all the tested cases. Moreover, GPS locations were accurately detected across all cases. Again a 100\% detection accuracy of spotting deviations from normal rates was achieved and it is illustrated in Table II.

\section{CONCLUSIONS AND FUTURE WORK}

In this paper, we presented a real-time monitoring system appropriate for users with coronary artery disease. The proposed system is based on a simple and inexpensive wearable sensor that detects heart rate and sends it wirelessly to a mobile device via an arduino microcontroller. A monitoring algorithm is applied on the mobile phone for checking variances from normal heart rates and raising an alarm to the closest hospital and the patients' relatives.

To prove the effectiveness of the proposed system, we carried out two experiments. In the first experiment, the system was tested on 20 healthy individuals for both rest and sport modes. In the second experiment, a simulation for 20 cases of patients with heart problems was conducted. In both experiments, the monitoring component provided a $100 \%$ detection accuracy. For the first experiment, no false alarms were generated and for the second experiment, the deviations from normal rates were detected and alarms were generated.

In the future, we plan to make the application cross-platform to be applicable on other platforms such as Android and IOS. Moreover, we plan to integrate the system with a diagnosis component for real-time diagnosis of the existence of CAD.

\section{REFERENCES}

[1] N. Oliver and F. F. Mangas, "HealthGear: A real-time wearable system for monitoring and analyzing physiological signals," presented at IEEE International Workshop on Wearable and Implantable Body Sensor Networks, Cambridge, USA, 2006.

[2] M. Sung, C. Marci, and A. Pentland, "Werable feecback systems for rehabilitation," Journal of Neuroengineering and Rehabilitation, vol. 2, no. 17, 2005.

[3] American Heart Association. (November 2014). [Online]. Available: http://www.heart.org/HEARTORG/

[4] R. Das, I. Turkoglu, and A. Sengur, "Effective diagnosis of heart disease through neural networks ensembles," Expert Systems with Applications, vol. 36, no. 4, pp. 7675-7680, 2004.

[5] H. Alemdar and C. Ersoy, "Wireless sensor networks for healthcare: A survey,” Computer Networks, vol. 54, no. 15, pp. 2688-2710, 2010.
[6] U. Anliker, J. A. Ward, P. Lukowicz et al., "AMON: A wearable multiparameter medical monitoring and alert system," IEEE Transactions on Information Technology in Biomedicine, vol. 8, pp. 415-427, 2004.

[7] S. Patel, H. Park, P. Bonato, L. Chan, and M. Rodgers, "A review of wearable sensors and systems with application in rehabilitation," Journal of Neuroengineering and Rehabilitation, vol. 9, no. 1, 2012.

[8] H. Asada, P. Shaltis, A. Reisner, S. Rhee, and R. Hutchinson, "Mobile monitoring with wearable photoplethysmographic biosensors," IEEE Engineering in Medicine and Biology Magazine, vol. 22, no. 3, pp. $28-40,2003$

[9] J. Patterson, D. G. McIlwraith, and G. Z. Yang, "A flexible, low noise reflective PPG sensor platform for ear-worn heart rate monitoring," in Proc. the IEEE Sixth International Workshop on Wearable and Implantable Body Sensor Networks, pp. 268-291, CA, USA, 2009.

[10] P. Pierleoni, L. Pernini, A. Belli and L. Palma, "An android-based heart monitoring system for the elderly and for patients with heart disease," International Journal of Telemedicine and Applications, vol. 2014, 2014.

[11] J. Habetha, "The MyHeart project-fighting cardiovascular diseases by prevention and early diagnosis," in Proc. the IEEE Eng Med Biol Soc. pp. 6746-6749, 2006.

[12] Pulse Sensor. (November 2014). [Online]. Available: http://pulsesensor.myshopify.com/

[13] A. Asuncion and D. Newman, UCI Machine Learning Repository, 2007.

Ahmed Otoom is currently working as an assistant professor at the Software Engineering Department at Hashemite University, Jordan. He has a $\mathrm{PhD}$ degree in computer science from the University of Technology, Sydney (UTS), Australia, in 2010. In 2003, he received his master's degree in software engineering from the University of Western Sydney, Australia. In 2002, he received his bachelor degree in computer science from Jordan University of Science and Technology, Jordan. His main research interests include computer vision and pattern recognition techniques for image and video analysis with a focus on realistic scenarios within video surveillance. $\mathrm{He}$ has also research interests in the bioinformatics area.

Ahmed Kefaye has finished his bachelor degree of computer science from the Hashemite University, Jordan. He is currently working as a system engineer at Alpha-Hub organization, Jordan. His main research interests include networking and wireless communications. He holds a number of certificates from Cisco organization.

Mohammad Ashour has recently finished his bachelor degree of computer information systems from the Hashemite University, Jordan. He is currently working as a software developer at Teleogx organization, Jordan. His main research interests include telecommunications, data mining and wireless communication.

Yousef Shanti has recently finished his bachelor degree of computer science from the Hashemite University, Jordan. $\mathrm{He}$ is currently working as a software developer at UXBERT organization, Saudi Arabia. His main research interests include human-computer interaction, computer vision and machine learning.

Mohammad Al-Majali has recently finished his bachelor degree of computer science from the Hashemite University, Jordan. He is currently working as a field officer at Niche marketing management organization, UAE. His main research interests include biomedical engineering and wireless communications. 